\title{
Evaluation of the analytical performance of a portable ion-selective electrode meter for measuring whole-blood, plasma, milk, abomasal-fluid, and urine sodium concentrations in cattle
}

\author{
A. A. Megahed, ${ }^{1,2}$ M. Hiew, ${ }^{3}$ W. Grünberg, ${ }^{4 *}$ F. M. Trefz,,${ }^{5}$ and P. D. Constable ${ }^{1} \dagger$ \\ ${ }^{1}$ Department of Veterinary Clinical Medicine, College of Veterinary Medicine, University of Illinois at Urbana-Champaign 61802 \\ ${ }^{2}$ Department of Animal Medicine (Internal Medicine), Faculty of Veterinary Medicine, Benha University Moshtohor-Toukh, Kalyobiya, Egypt 13736 \\ ${ }^{3}$ Department of Veterinary Clinical Studies, Faculty of Veterinary Medicine, Universiti Putra, Selangor, Malaysia 43400 \\ ${ }^{4}$ Department of Veterinary Clinical Sciences, Purdue University, College of Veterinary Medicine, West Lafayette, IN 47907 \\ ${ }^{5}$ Clinic for Ruminants with Ambulatory and Herd Health Services at the Center of Veterinary Clinical Medicine, LMU Munich, \\ 85764 Oberschleißheim, Germany
}

\section{ABSTRACT}

A portable ion-selective electrode (ISE) meter (LAQUAtwin B-722; Horiba Instruments Inc., Irvine, CA) is available for measuring the sodium ion concentration $([\mathrm{Na}])$ in biological fluids. The objective of this study was to characterize the analytical performance of the ISE meter in measuring [Na] in whole-blood, plasma, milk, abomasal fluid, and urine samples from cattle. Method comparison studies were performed using whole-blood and plasma samples from 106 sick calves and 11 sick cows admitted to a veterinary teaching hospital, 80 milk and 206 urine samples from 16 lactating Holstein-Friesian cows with experimentally induced free water, electrolyte, and acid-base imbalances, and 67 abomasal fluid samples from 7 healthy male Holstein-Friesian calves fed fresh milk with or without an oral electrolyte solution. Deming regression and Bland-Altman plots were used to determine the accuracy of the meter against reference methods. The meter used in direct mode on undiluted samples measured whole-blood [Na] $9.7 \mathrm{mmol} / \mathrm{L}(7.3 \%)$ lower than a direct ISE reference method and plasma [Na] 16.7 $\mathrm{mmol} / \mathrm{L}(12.7 \%)$ lower than an indirect ISE reference method. The meter run in direct mode measured milk [Na] $3.1 \mathrm{mmol} / \mathrm{L}$ lower and abomasal fluid [Na] $9.0 \%$ lower than indirect ISE reference methods. The meter run in indirect mode on diluted samples accurately measured urine $[\mathrm{Na}]$ compared with an indirect ISE reference method. We conclude that, after adjustment

Received December 21, 2018.

Accepted April 23, 2019.

* Current address: University of Veterinary Medicine Hannover, Foundation, Clinic for Cattle, Bischofsholer Damm 15, 30173 Hanover, Germany.

†Corresponding author: constabl@illinois.edu for the bias determined from Bland-Altman plots, the LAQUAtwin ISE meter provides a clinically useful and low-cost cow-side instrument for measuring $[\mathrm{Na}]$ in whole blood, plasma, milk, and abomasal fluid.

Key words: hyponatremia, hypernatremia, point-ofcare, cattle

\section{INTRODUCTION}

Ion-selective electrode (ISE) technology is widely used to analyze cation and selected anion concentrations in biological fluids because the method is accurate, rapid, and inexpensive (Burnett et al., 2000; D'Orazio et al., 2000). The ISE method measures electrolyte activity in the aqueous portion of the fluid and is currently applied using either of 2 approaches: direct ISE of an undiluted sample, or indirect ISE of a diluted sample. Direct ISE methods are commonly used in point-of-care devices, and, when used in whole blood, plasma and serum, or milk, the measured electrolyte concentration is not affected by the plasma protein concentration or the milk fat and protein percentage, respectively (Megahed et al., 2016). In contrast, indirect ISE methods are widely used in automated analyzers in diagnostic laboratories because they require a lower sample volume and provide a larger analytical range, permitting automated measurement of electrolyte activity in biological fluids such as urine, saliva, and sweat, as well as plasma or serum (Dimeski et al., 2012). However, indirect ISE methods can result in underestimation of the plasma or serum electrolyte concentration in hyperproteinemic and hyperlipidemic samples and overestimation of electrolyte concentrations in hypoproteinemic samples (D'Orazio et al., 2000; Dimeski and Barnett, 2005; Dimeski et al., 2006; Jain et al., 2009) as a result of sample dilution. Direct ISE methods, when appropriately calibrated, are considered superior to indirect ISE methods for the measurement of electrolytes in blood, plasma, and 
serum, because the measured value is not affected by the plasma protein or lipid concentration.

A portable ISE meter (LAQUAtwin B-722; Horiba Instruments Inc., Irvine, CA) has recently become available for measuring the sodium concentration ([Na]) in biological fluids. The low cost $(\$ 350)$ and portability of the meter make it potentially useful for the on-farm measurement of $[\mathrm{Na}]$ in whole blood, plasma, milk, urine, and other fluids from adult cattle and calves. Once purchased, the low cost of calibration solutions results in an extremely low cost per test (approximately $\$ 0.02-\$ 0.03$ per test). We hypothesized that the LAQUAtwin ISE meter would provide a clinically relevant and practical method for measuring $[\mathrm{Na}]$ in whole blood, plasma, milk, urine, and abomasal fluid from cattle. The objective of this study was to apply recommended statistical methods for methods comparison studies to characterize the analytical performance of the LAQUAtwin ISE meter in measuring $[\mathrm{Na}]$ in these fluid samples.

\section{MATERIALS AND METHODS}

\section{Calibration and Measurement Resolution of the LAQUAtwin ISE Meter}

The LAQUAtwin B-722 $\mathrm{Na}^{+}$meter was used in direct mode (no dilution of the sample) or indirect mode (dilution of the sample). The meter was calibrated before analysis using a 2-point calibration and standard $\mathrm{Na}$ solutions with $150 \mathrm{ppm}(6.5 \mathrm{mmol} / \mathrm{L})$ and 2,000 ppm $(86 \mathrm{mmol} / \mathrm{L})$, provided by the manufacturer. The calibration solutions were selected because they were recommended by the manufacturer and within the recommended range of measurement. The displayed value in ppm (equivalent to $\mathrm{mg} / \mathrm{L}$ ) was multiplied by 0.043 (the reciprocal of the molecular weight of sodium = $23.0 \mathrm{~g} / \mathrm{mol}=23.0 \mathrm{mg} / \mathrm{mmol}$ ) to obtain the SI unit $\mathrm{mmol} / \mathrm{L}$. The meter had a total display range of $(0$ to 99$) \times 100 \mathrm{ppm}$ in 3 automatically switched ranges: the $\times 1$ range $(1$ to $99 \mathrm{ppm}=0.043$ to $4.3 \mathrm{mmol} / \mathrm{L}$; resolution, $1 \mathrm{ppm}=0.043 \mathrm{mmol} / \mathrm{L})$, the $\times 10$ range (100 to $990 \mathrm{ppm}=4.3$ to $43.0 \mathrm{mmol} / \mathrm{L}$; resolution, 10 $\mathrm{ppm}=0.43 \mathrm{mmol} / \mathrm{L})$, and the $\times 100$ range $(1,000$ to $9,900 \mathrm{ppm}=43$ to $430 \mathrm{mmol} / \mathrm{L}$; resolution, $100 \mathrm{ppm}$ $=4.3 \mathrm{mmol} / \mathrm{L})$. The meter's display blinked whenever the fluid [Na] exceeded 2,300 ppm (99 mmol/L); consequently, the manufacturer recommends that the displayed value should be considered only as an approximation when $[\mathrm{Na}$ ] ranged from 2,300 to 9,900 ppm (99 to $426 \mathrm{mmol} / \mathrm{L}$ ). The manufacturer reported repeatability as $\pm 10 \%$ over a $\mathrm{pH}$ range of 3 to 9 , and, where noted, samples with $\mathrm{pH}<3.0$ or $>9.0$ were not analyzed. Selectivity coefficients (concentration ratio of the interfering ion against the target ion) to other monovalent or divalent cations were reported as follows: $\mathrm{K}^{+}$, $\mathrm{Rb}^{+}=1 \times 10^{-2} ; \mathrm{Ba}^{2+}, \mathrm{Sr}^{2+}, \mathrm{Ca}^{2+}, \mathrm{Mg}^{2+}=1 \times 10^{-4}$; $\mathrm{Li}^{+}=1 \times 10^{-3} ; \mathrm{Cs}^{+}=3 \times 10^{-3} ; \mathrm{NH}_{4}^{+}=6 \times 10^{-3}$. The meter corrected the ISE measurement for temperature, and the operating temperature range was reported to be $5^{\circ} \mathrm{C}$ to $40^{\circ} \mathrm{C}$ at up to $85 \%$ relative humidity.

\section{Measurement of Whole-Blood and Plasma Sodium Concentration}

Whole-blood samples were collected from 117 animals (11 cows and 106 calves) admitted to the Clinic for $\mathrm{Ru}-$ minants, LMU Munich (Oberschleißheim, Germany), between November 2016 and April 2017, for a variety of medical conditions. Most calves and cows were of Fleckvieh breed $(\mathrm{n}=103,88 \%)$. Clinical management of cows and calves was based on standardized clinic protocols, and, consequently, approval from an animal ethics committee was not required. Blood samples were anaerobically collected from the jugular vein into lithium heparin tubes on admission. Whole-blood $\mathrm{pH}$ and $[\mathrm{Na}]$ were first measured using a blood gas analyzer (Rapidpoint 405, Siemens Healthcare Diagnostics Inc., Tarrytown, NY), which provided a direct measure of $[\mathrm{Na}]$, as samples were not diluted for analysis. Immediately thereafter, whole-blood $[\mathrm{Na}]$ was measured in the same sample, using a portable ISE meter in direct mode according to the manufacturer's recommendations.

The remainder of the heparinized whole-blood sample was centrifuged for $5 \mathrm{~min}$ at $1,400 \times g$ at room temperature with tube capped to minimize evaporation of water that would result in increased plasma [Na] (Bijster et al., 1983). Plasma was harvested immediately after centrifugation, and plasma $[\mathrm{Na}]$ was measured using the same ISE meter in the same manner as for wholeblood analysis. The time interval between measuring whole-blood and plasma [Na] was always $<30 \mathrm{~min}$. The remainder of the plasma sample was transferred to a polypropylene vial and submitted to an in-house laboratory, where plasma [Na] was measured using an automatic analyzer as described below.

\section{Measurement of Milk Sodium Concentration}

We collected 5 milk samples from each of 16 lactating Holstein-Friesian cows with experimentally induced free water, electrolyte, and acid-base imbalances, as described by Constable et al. (2014). Milk samples were stored at $-20^{\circ} \mathrm{C}$ for up to 2 years and thawed at room temperature and vortexed. Milk $[\mathrm{Na}]$ was measured directly, using the portable ISE meter, in the 
same manner as described previously for whole blood and plasma. Visible globules of fat were avoided when milk was aspirated from the sample and transferred to the sensor pad. Centrifugation of the milk sample and removal of the fat layer was not performed, because the ISE analyzer was being evaluated as a cow-side test.

\section{Measurement of Abomasal Fluid Sodium Concentration}

A total of 94 abomasal fluid samples were collected from 7 healthy male Holstein-Friesian calves via surgically placed abomasal cannulas as described by Constable et al. (2009). Of the 7 calves, 1 completed only 2 of the treatment arms, and data from this calf are included here. Calves were fed fresh cow milk (approximately $3.5 \%$ fat, $3.4 \%$ protein) with or without the addition of 1 of 2 oral rehydration solutions that contained $\mathrm{Na}$. Abomasal fluid samples were stored at $-20^{\circ} \mathrm{C}$ for up to 6 years. Of the 94 samples, 27 were collected when abomasal $\mathrm{pH}<3.0$; the manufacturer reports that sample $\mathrm{pH}<3.0$ can influence the measured [Na]. Consequently, Na measurements were confined to the 67 samples obtained where abomasal fluid $\mathrm{pH}>3.0$. Samples were thawed at room temperature, vortexed, and $[\mathrm{Na}]$ measured directly, using the portable ISE meter, in the same manner as described previously for whole blood and plasma.

\section{Measurement of Urine Sodium Concentration}

A total of 206 urine samples were collected from 16 lactating Holstein-Friesian cows with experimentally induced free water, electrolyte and acid-base imbalances, as described by Constable et al. (2014). Thirteen samples were obtained from each of 15 cows, and 11 samples were obtained from the remaining cow. Urine $\mathrm{pH}$ was measured immediately, using a test strip that measured urine $\mathrm{pH}$ in 0.5-unit increments (Siemens Diagnostics 2181 Labstix Reagent Strips; Siemens Medical Solutions USA Inc., Malvern, PA). Urine samples were stored at $-20^{\circ} \mathrm{C}$ for up to 2 years, thawed at room temperature, vortexed, and diluted 1:19 with distilled water to minimize the potential for ion-binding effects of low-molecular-weight zwitterions present in bovine urine (Brooks et al., 1988; Megahed et al., 2016) and because of the anticipated large range in values for urine $[\mathrm{Na}]$. The $[\mathrm{Na}]$ of diluted urine (indirect mode) was measured, using the ISE meter in the same manner as described previously for whole blood and plasma, and the measured value was corrected for dilution. Collection of milk, urine, and abomasal fluid samples was approved by the Purdue Animal Care and Use Committee.

\section{Reference Methods for Measuring Plasma, Milk, Abomasal Fluid, and Urine Sodium Concentration}

Plasma samples were analyzed at the Clinic for $\mathrm{Ru}$ minants, LMU Munich (Oberschleißheim, Germany) using an indirect ion-selective potentiometry automatic analyzing system (Cobas c 311, Roche Diagnostics, Mannheim, Germany). The Cobas c 311 analyzer was also used to determine the plasma total protein concentration (Biuret method).

Milk, abomasal fluid, and urine samples were analyzed at the Veterinary Diagnostic Laboratory at the University of Illinois at Urbana-Champaign, using an indirect ISE method and 1 of 2 automated analyzers (milk, urine: Hitachi 917, Roche, Basel, Switzerland; abomasal fluid: Hitachi 911, Roche). Milk samples were centrifuged and the fat layer removed before being analyzed using the reference method. The automated analyzers used a liquid/liquid junction type of ISE and diluted the sample 1:31 with boric acid $(650 \mathrm{mmol} / \mathrm{L})$ to acidify the sample before analysis; consequently, the reference methods for milk, urine, and abomasal fluid $[\mathrm{Na}]$ were based on indirect potentiometry, with a standard correction factor applied by the instrument to ensure equivalence with flame photometry.

\section{Statistical Analysis}

Statistical analyses were performed using MedCalc Statistical Software version 18.11 (MedCalc Software bvba, Ostend, Belgium, 2018) and SAS 9.4 (SAS Inc., Cary, NC). Statistical significance was set at $P<0.05$. A minimum sample size of 40 over a wide physiologic range of values is recommended in method comparison studies, although larger sample sizes are recommended for analytes with a low range ratio, which represents the maximum value divided by the minimum value (Jensen and Kjelgaard-Hansen, 2006). We identified a minimum sample size of 40 as an achievable goal based on stored milk, abomasal fluid, and urine samples and access to whole-blood and plasma samples. Sample sizes larger than 40 were used when possible. Within-day repeatability was evaluated by calculating the coefficient of variation (CV) from 20 consecutive analyses of a purposively collected sample on the same day. Clinical performance of the portable analyzer was assessed by comparison to the allowable total error $\left(\mathbf{T E}_{\mathrm{a}}\right)$, which sets the limit for combined imprecision (random error) and bias (systematic error) that is acceptable for clinical purposes when measuring $[\mathrm{Na}]$ in a single measurement (Harr et al., 2013). The value for $\mathrm{TE}_{\mathrm{a}}$ was assigned as reference $[\mathrm{Na}] \pm 4 \mathrm{mmol} / \mathrm{L}$ for blood and plasma (Ehrmeyer et al., 1990), or reference $[\mathrm{Na}] \pm 5 \%$ for milk, abomasal fluid, and urine (Harr et al., 2013). 
Deming regression was used to assess the linear relationship between whole-blood and plasma $[\mathrm{Na}]$ measured by the portable ISE meter, as well as between whole-blood, plasma, milk, urine, and abomasal fluid [Na] measured by the ISE meter and reference methods (Martin, 2000), using the within-day CV value for the middle of the range for each analytical method and assuming a similar CV value for the reference method. For Deming regression, the intercept value reflects constant bias, and the slope reflects proportional bias.

Bland-Altman difference plots were constructed as the primary method to characterize agreement between the ISE meter and the reference method. The mean bias and associated $95 \%$ confidence interval for its estimate were calculated for analyses of blood or plasma from the associated standard error (Giavarina, 2015); the bias estimate reflects the mean bias over the range of measured values and therefore includes both the constant and proportional bias identified using Deming regression. Linear regression was used to investigate the presence of proportional error in BlandAltman plots where single measurements were obtained per individual (whole-blood and plasma samples), with the differences regressed against the mean values (Giavarina, 2015). The presence of proportional error was investigated by visual inspection of the plot when multiple measurements were obtained per individual (milk, abomasal fluid, and urine samples). Upper and lower limits of agreement (LoA) in Bland-Altman plots were calculated from the mean bias $\pm 1.96 \times$ the standard deviation. The plot was visually examined to determine whether differences were symmetrically distributed around 0 (homoscedastic) and to verify that $95 \%$ of the differences were between the upper and lower LoA (Jensen and Kjelgaard-Hansen, 2006). The 95\% confidence interval for the limits of agreement (95\% CI for LoA) was calculated using Zou's method of variance estimates recovery (Bland and Altman, 2007; Zou, 2013; Olofsen et al., 2015); this method is appropriate when single measurements per individual (whole-blood and plasma samples) or multiple measurements per individual (milk, abomasal fluid, and urine samples) are obtained. The ISE meter was considered suitable for measuring $[\mathrm{Na}]$ in the sample matrix when the $95 \%$ CI for LoA $<\mathrm{TE}_{\mathrm{a}}$ (Chhapola et al., 2015).

\section{RESULTS}

\section{Within-Day Repeatability}

The within-day CV for whole-blood samples with [Na] of 97,132 , and $160 \mathrm{mmol} / \mathrm{L}$ measured by the portable ISE meter were $2.5 \%, 2.5 \%$, and $2.0 \%$, respectively.
Mean within-day CV for plasma samples measured by the portable ISE meter with [Na] of 92, 122, and 153 $\mathrm{mmol} / \mathrm{L}$ were $2.9 \%, 2.8 \%$, and $2.2 \%$, respectively. The within-day $\mathrm{CV}$ for milk samples with $[\mathrm{Na}]$ measured by the portable ISE meter of 13,19 , and $27 \mathrm{mmol} / \mathrm{L}$ were $3.7 \%, 3.8 \%$, and $3.2 \%$, respectively. The within-day CV for abomasal fluid samples with [Na] of 15, 41, and $79 \mathrm{mmol} / \mathrm{L}$ measured by the portable ISE meter were $2.1 \%, 2.3 \%$, and $4.8 \%$, respectively. The within-day CV for urine samples with [Na] of 12,77 or $121 \mathrm{mmol} / \mathrm{L}$ measured by the portable ISE meter were $6.6 \%, 4.4 \%$, and $3.9 \%$, respectively.

\section{Whole-Blood and Plasma Sodium Concentration}

A linear relationship existed between whole-blood [Na] measured using the portable ISE meter and the Rapidpoint 405 blood analyzer for 117 whole-blood samples (Figure 1A), with both measurements made in direct mode. Deming regression indicated absence of proportional bias (slope $=0.99 ; 95 \%$ CI: 0.93 to 1.03) but presence of a constant bias (intercept $=-7.6$ $\mathrm{mmol} / \mathrm{L} ; 95 \%$ CI: -17.7 to -0.5$)$. The Bland-Altman plot indicated that the bias for the ISE meter was -9.7 $\mathrm{mmol} / \mathrm{L}(95 \% \mathrm{CI}:-10.4$ to $-9.0 \mathrm{mmol} / \mathrm{L} ; P<0.001$ compared with 0) when compared with the Rapidpoint 405 blood analyzer. The 95\% LoA ranged from -17.0 (95\% CI: -18.2 to -15.8$)$ to $-2.3(95 \%$ CI: -3.5 to $-1.1) \mathrm{mmol} / \mathrm{L}$. Inspection of the plot indicated that the differences were homoscedastic, and approximately 95\% of the data points were within the LoA (Figure 1B). Proportional error was not present in the plot, based on the estimated slope value for linear regression of differences against mean values (slope $=0.037 ; P=$ 0.49). The $95 \%$ CI for LoA, representing the difference between -18.2 and $-1.1 \mathrm{mmol} / \mathrm{L}$, was $17.1 \mathrm{mmol} / \mathrm{L}$. In summary, the bias was significantly lower than 0 , and the $95 \%$ CI for the LoA exceeded the a priori defined range of $8.0 \mathrm{mmol} / \mathrm{L}$.

A linear relationship existed between whole-blood and plasma $[\mathrm{Na}]$ measured by the portable ISE meter in 117 samples (Figure 2A), with both measurements made in direct mode. Deming regression indicated absence of proportional bias (slope $=0.95 ; 95 \%$ CI: 0.88 to 1.01) but presence of a constant bias (intercept $=11.5$ mmol/L; 95\% CI: 3.0 to 20.0). The Bland-Altman plot indicated that bias for the ISE meter was $+4.7 \mathrm{mmol} / \mathrm{L}$ (95\% CI: 4.1 to $5.4 \mathrm{mmol} / \mathrm{L} ; P<0.001$ compared with 0 ) when reading whole blood compared with plasma. The $95 \%$ LoA ranged from -2.2 (95\% CI: -3.3 to -1.1$)$ to 11.7 (95\% CI: 10.6 to 12.8 ) $\mathrm{mmol} / \mathrm{L}$. Inspection of the plot indicated that the differences were homoscedastic, and approximately $95 \%$ of the data points were 
within the LoA (Figure 2B). Proportional error was not present in the plot, based on the estimated slope value for linear regression of differences against mean values (slope $=-0.052 ; P=0.12$ ). The $95 \%$ CI for LoA was $16.1 \mathrm{mmol} / \mathrm{L}$. In summary, the bias was significantly greater than 0 , and the $95 \%$ CI for the LoA exceeded the a priori defined range of $8.0 \mathrm{mmol} / \mathrm{L}$.
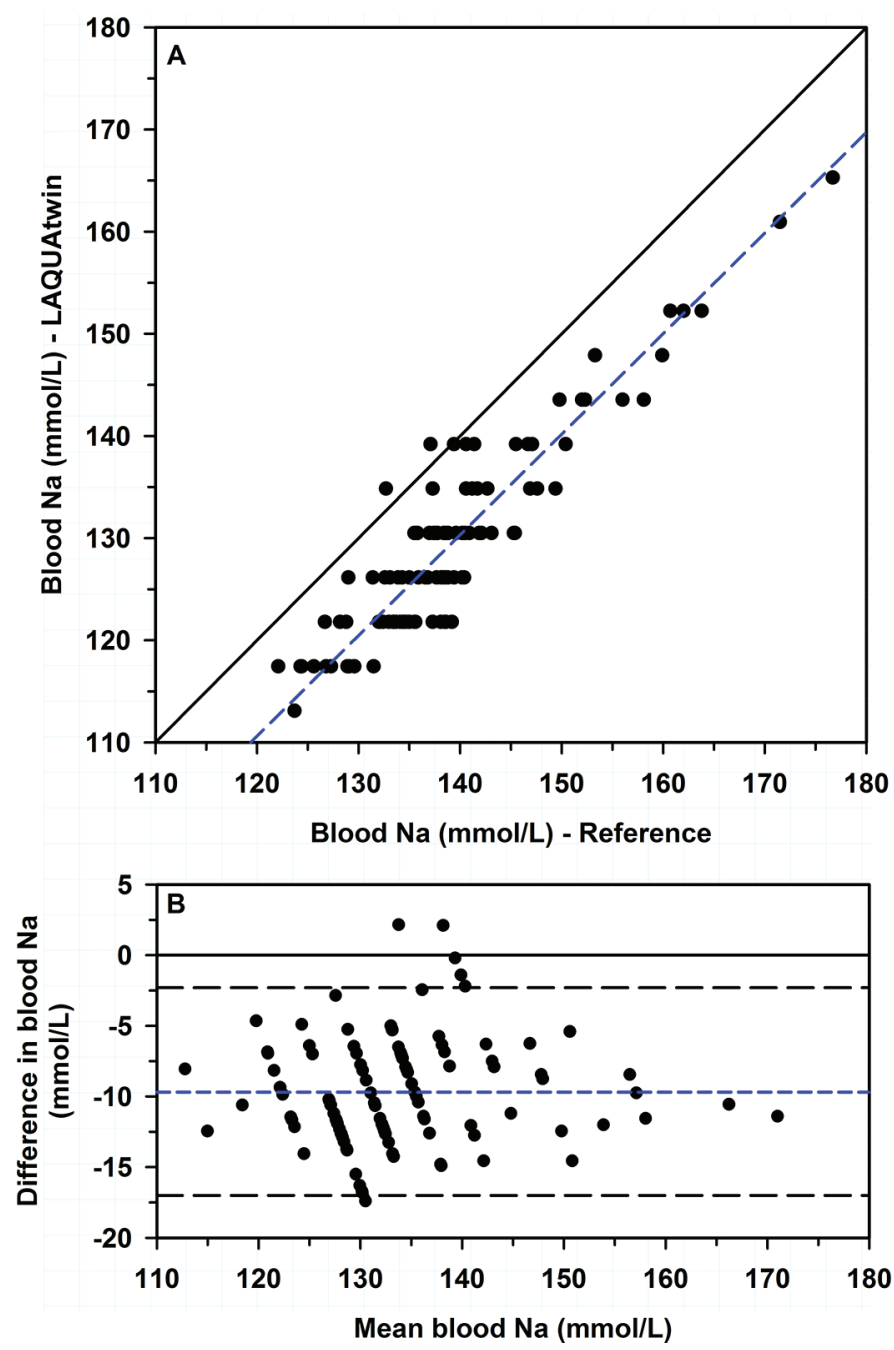

Figure 1. (A) Scatterplot indicating the relationship between whole-blood sodium concentration ([Na]) measured by an ion-selective electrode (ISE) method (LAQUAtwin $\mathrm{Na}^{+}$meter; Horiba Instruments Inc., Irvine, CA) versus the whole-blood [Na] measured by Rapidpoint blood gas point-of-care unit (Siemens Healthcare Diagnostics Inc., Tarrytown, NY) for 117 whole-blood samples. Many data points are superimposed because the LAQUAtwin meter has a resolution of 4.3 $\mathrm{mmol} / \mathrm{L}$ when $[\mathrm{Na}]>43 \mathrm{mmol} / \mathrm{L}$. The solid diagonal line is the line of identity, and the blue dashed line is the line of best fit from Deming regression. (B) Bland-Altman mean difference plot. The horizontal blue line with short dashes represents the mean bias, and the horizontal lines with long dashes reflect the $95 \%$ limits of agreement. The plot indicates that the ISE meter measured whole-blood [Na] $9.7 \mathrm{mmol} / \mathrm{L}$ $(7.3 \%)$ lower than the Rapidpoint blood gas analyzer when both analyzers were run in direct (undiluted) mode.
Deming regression for plasma $[\mathrm{Na}]$ measured by the portable ISE meter in direct mode against plasma $[\mathrm{Na}]$ measured by the indirect reference method (Cobas c 311) for 117 plasma samples indicated a proportional bias (1.06; 95\% CI: 0.92 to $1.20 \mathrm{mmol} / \mathrm{L})$ that was not different from 1 and a constant bias $(-25.1 \mathrm{mmol} / \mathrm{L}$; $95 \% \mathrm{CI}:-44.2$ to $-6.0 \mathrm{mmol} / \mathrm{L})$ that was different from 0 (Figure 3A). The Bland-Altman plot indicated that the bias for the ISE meter run in direct mode was
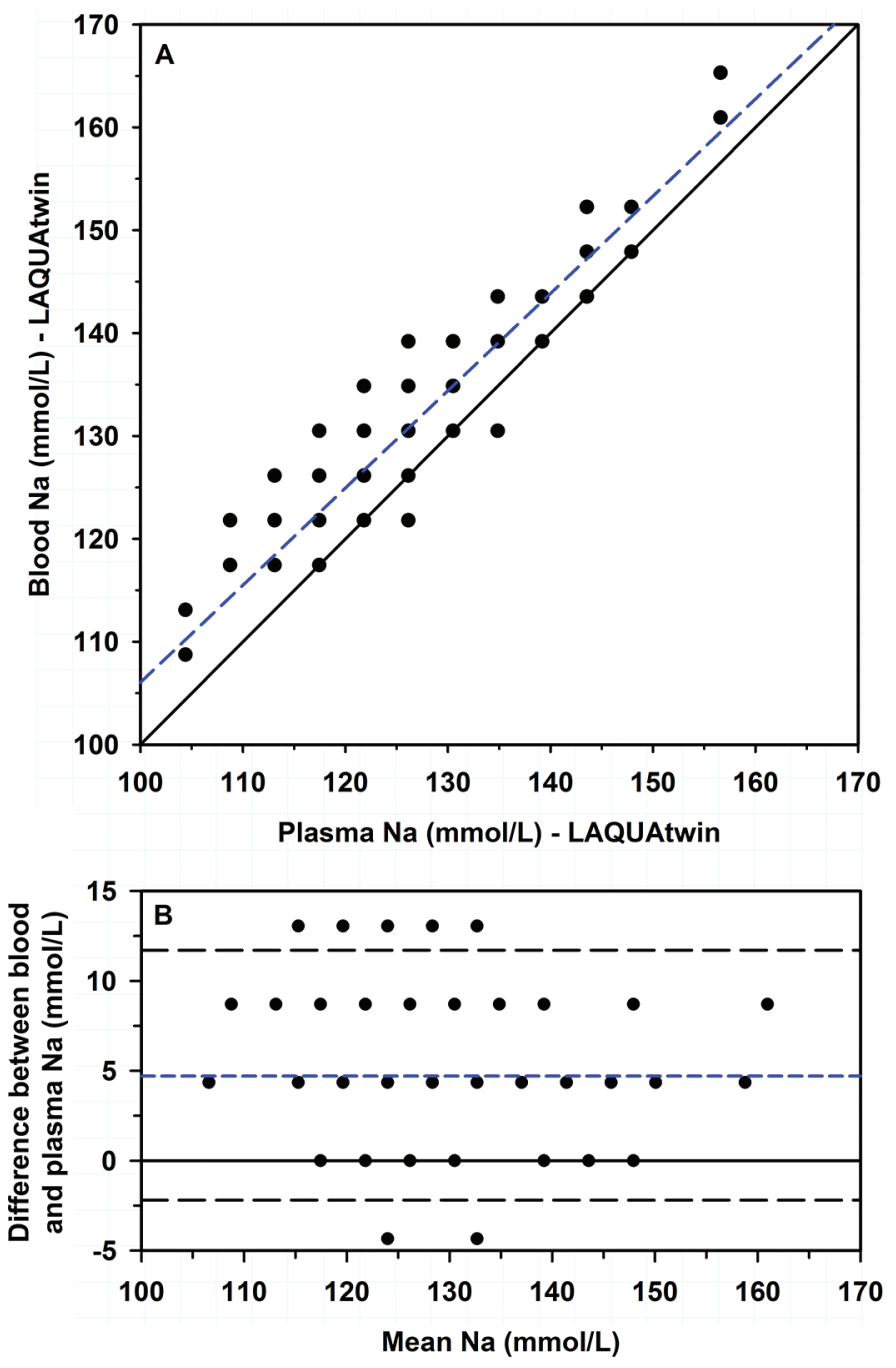

Figure 2. (A) Scatterplot indicating the relationship between whole-blood and plasma sodium concentration ([Na]) determined by an ion-selective electrode (ISE) method (LAQUAtwin $\mathrm{Na}^{+}$meter; Horiba Instruments Inc., Irvine, CA) for 117 samples. Many data points are superimposed because the meter has a resolution of $4.3 \mathrm{mmol} / \mathrm{L}$ when $[\mathrm{Na}]>43 \mathrm{mmol} / \mathrm{L}$. The solid diagonal line is the line of identity, and the blue dashed line is the line of best fit from Deming regression. (B) Bland-Altman difference plot. The horizontal blue line with short dashes represents the mean bias, and the horizontal lines with long dashes reflect the $95 \%$ limits of agreement. The plot indicates that the ISE meter measured whole-blood [Na] $4.7 \mathrm{mmol} / \mathrm{L}(3.8 \%)$ higher than plasma $[\mathrm{Na}]$ when both were run in direct (undiluted) mode. 
$-16.7 \mathrm{mmol} / \mathrm{L}(95 \% \mathrm{CI}:-17.7$ to $-15.7 \mathrm{mmol} / \mathrm{L} ; P$ $<0.001$ compared with 0) compared with the indirect reference method. The 95\% LoA ranged from -27.4 (95\% CI: -29.1 to -25.7$)$ to -5.9 (95\% CI: -7.6 to $-4.2) \mathrm{mmol} / \mathrm{L}$. Inspection of the plot indicated that the differences were homoscedastic, and approximately $95 \%$ of the data points were within the LoA (Figure 3B). Proportional error was not present in the plot, based on the estimated slope value for linear regression of differences against mean values (slope $=0.034 ; P=$ 0.52). The $95 \%$ CI for LoA was $13.5 \mathrm{mmol} / \mathrm{L}$. In summary, the bias was significantly lower than 0 , and the 95\% CI for the LoA exceeded the a priori defined range of $8.0 \mathrm{mmol} / \mathrm{L}$.

\section{Milk Sodium Concentration}

Deming regression for milk $[\mathrm{Na}]$ measured by the portable ISE meter in a direct mode against milk [Na] measured by the indirect ISE reference method for 80 milk samples indicated a proportional bias $(0.88 ; 95 \%$ CI: 0.81 to 0.95$)$ that was less than 1 and constant bias $(-0.5 \mathrm{mmol} / \mathrm{L} ; 95 \%$ CI: -2.0 to 1.0$)$ that did not differ from 0 (Figure 4A). The Bland-Altman plot indicated that the ISE meter run in direct mode measured milk $[\mathrm{Na}] \quad 15.0 \%$ lower than the indirect ISE reference method $(P<0.001$ compared with 0$)$, with $95 \%$ limits of agreement from -24.1 to $-5.9 \%$ (Figure 4B).

The Bland-Altman plot indicated that the bias for the ISE meter run in direct mode was $-3.1 \mathrm{mmol} / \mathrm{L}$ compared with the indirect reference method. The $95 \%$ LoA ranged from -5.2 (95\% CI: -5.9 to -4.7$)$ to -1.0 (95\% CI: -1.4 to -0.3$) \mathrm{mmol} / \mathrm{L}$. Inspection of the plot indicated that the differences were homoscedastic, and approximately $95 \%$ of the data points were within the LoA (Figure 4B). Proportional error was not evident in the plot. The $95 \%$ CI for LoA was $5.6 \mathrm{mmol} / \mathrm{L}$. In summary, the bias was lower than 0 , and the $95 \%$ CI for the LoA exceeded the a priori defined range of \pm $4.0 \mathrm{mmol} / \mathrm{L}$.

\section{Abomasal Fluid Sodium Concentration}

Sixty-seven data points where abomasal fluid $\mathrm{pH}$ $>3.0$ were available for analysis. Deming regression for abomasal fluid $[\mathrm{Na}]$ measured by the portable ISE meter in a direct mode against abomasal fluid [Na] measured by the ISE reference method revealed a linear relationship (Figure 5A), with a slope of 0.90 (95\% CI: 0.88 to 0.93 ) that was less than 1 , and an intercept of $0.7 \mathrm{mmol} / \mathrm{L}(95 \% \mathrm{CI},-0.8$ to 2.2$)$ that was not different from 0 .

The Bland-Altman plot indicated that the bias for the ISE meter run in direct mode was $-8.6 \%$ compared with the indirect reference method. The 95\% LoA ranged from $-20.7 \%$ (95\% CI: -24.1 to -18.5$)$ to $5.4 \%$ (95\% CI: 2.6 to 10.1). Inspection of the plot indicated that the differences were homoscedastic, and all of the data points were within the LoA (Figure 5B). Proportional error was not evident in the plot. The $95 \%$ CI for LoA was $34.2 \%$. In summary, the bias was significantly
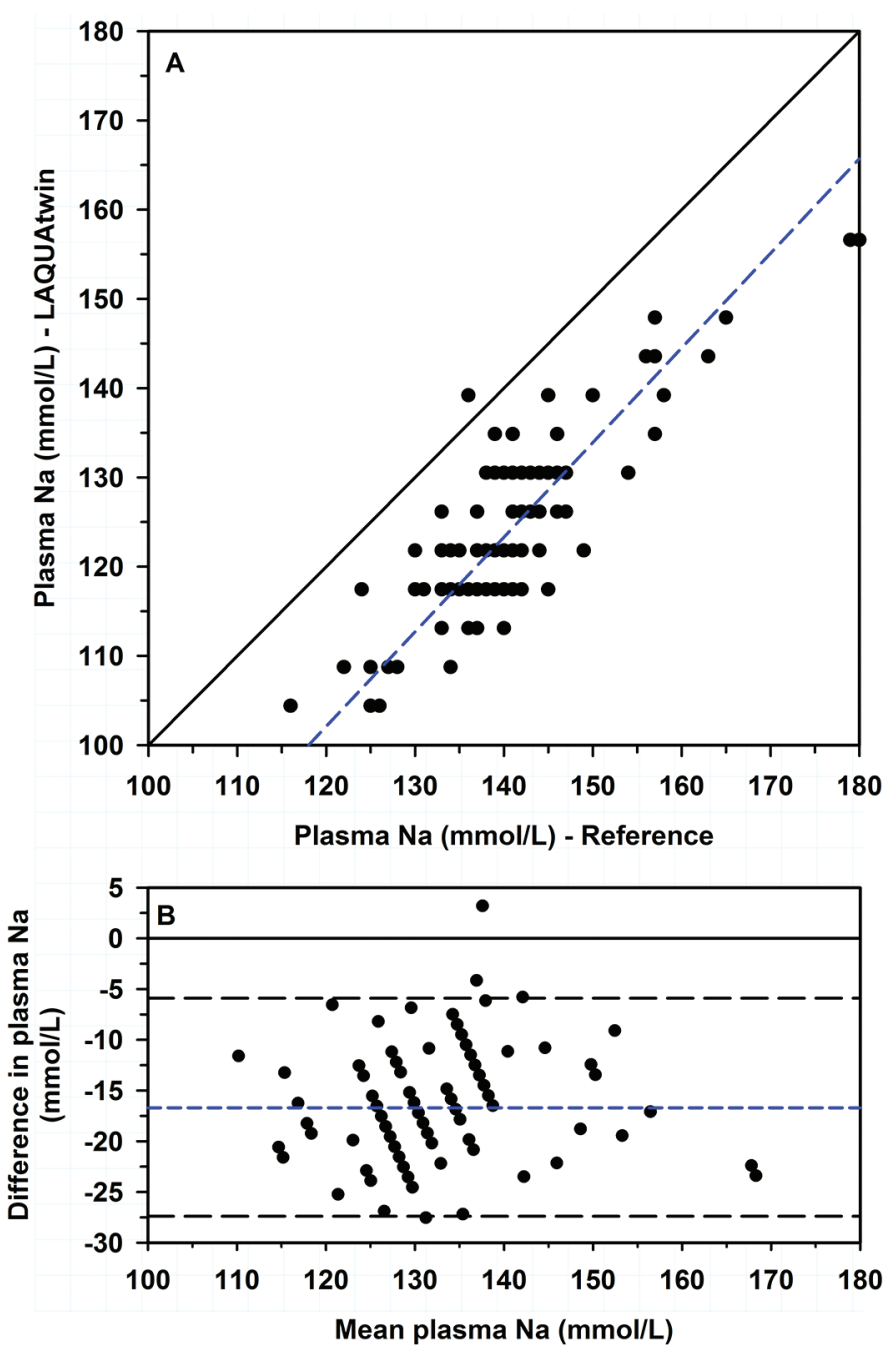

Figure 3. (A) Scatterplot indicating the relationship between the plasma sodium concentration ([Na]) measured by an ion-selective electrode (ISE) method in direct mode (LAQUAtwin $\mathrm{Na}^{+}$meter; Horiba Instruments Inc., Irvine, CA) versus the plasma [Na] measured by the reference method (indirect ISE) for 117 plasma samples. Many data points are superimposed because the LAQUAtwin meter has a resolution of $4.3 \mathrm{mmol} / \mathrm{L}$ when $[\mathrm{Na}]>43 \mathrm{mmol} / \mathrm{L}$. The solid diagonal line is the line of identity, and the blue dashed line is the line of best fit from Deming regression. (B) Bland-Altman mean difference plot. The horizontal blue line with short dashes represents the mean bias, and the horizontal lines with long dashes reflect the $95 \%$ limits of agreement. The plot indicates that the ISE meter run in direct mode measured plasma [Na] $16.7 \mathrm{mmol} / \mathrm{L}(12.6 \%)$ lower than the indirect ISE reference method. 

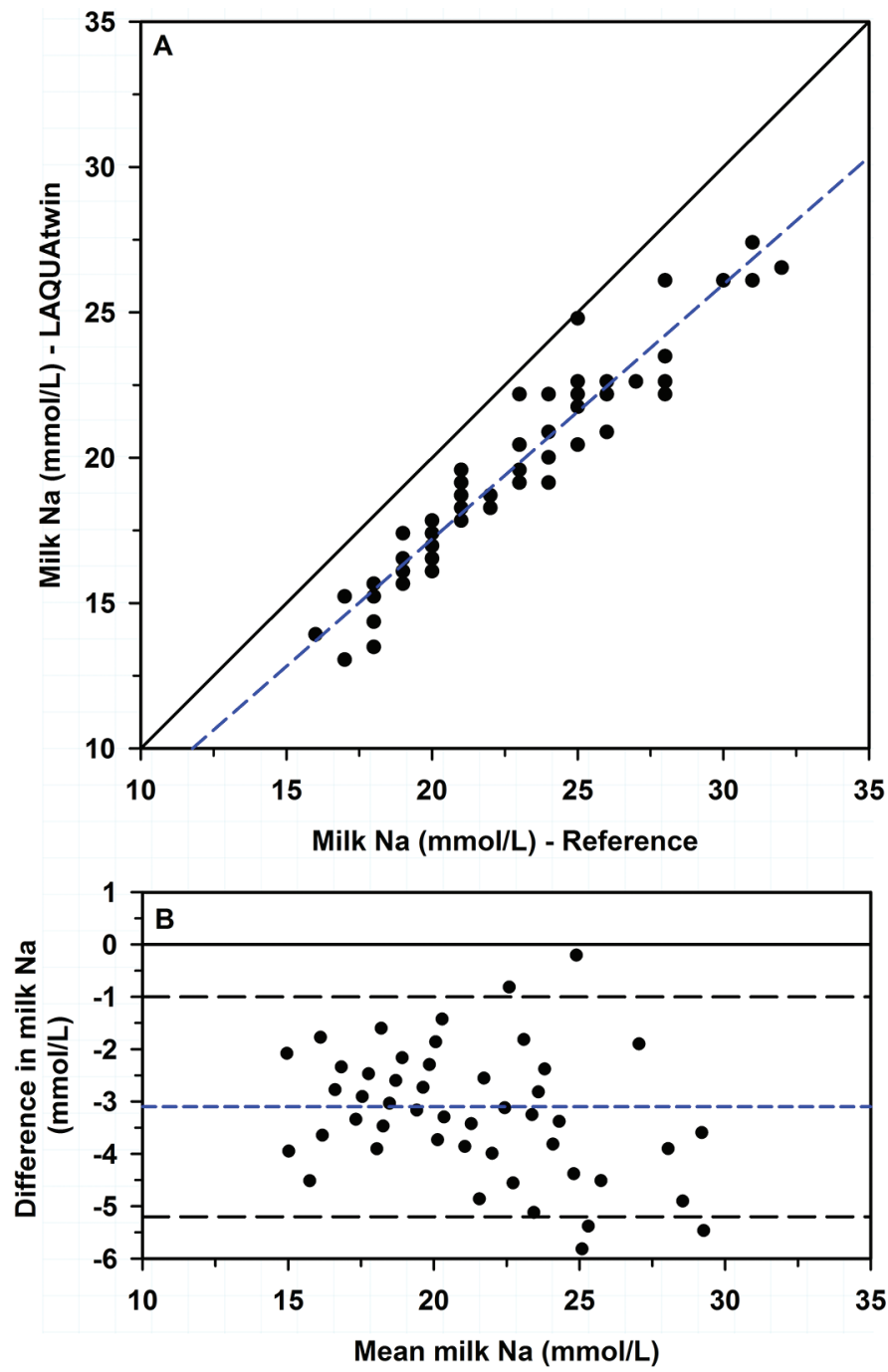

Figure 4. (A) Scatterplot indicating the relationship between the milk sodium concentration $([\mathrm{Na}])$ measured by a direct ion-selective electrode (ISE) method (LAQUAtwin $\mathrm{Na}^{+}$meter; Horiba Instruments Inc., Irvine, $\mathrm{CA}$ ) versus the milk [Na] measured by an indirect ISE reference method (1:31 dilution in distilled water). Milk samples $(\mathrm{n}=80)$ were obtained periodically from 16 lactating Holstein-Friesian cows with experimentally induced electrolyte and acid-base imbalances. The solid diagonal line is the line of identity, and the blue dashed line is the line of best fit from Deming regression. (B) Bland-Altman mean difference plot. The horizontal blue line with short dashes represents the mean bias, and the horizontal lines with long dashes reflect the $95 \%$ limits of agreement. The plot indicates that the ISE meter run in direct mode measured milk [Na] $3.1 \mathrm{mmol} / \mathrm{L}$ lower than the reference method.

lower than 0 , and the $95 \%$ CI for the LoA exceeded the a priori defined range of $\pm 5 \%$.

\section{Urine Sodium Concentration}

Urine $\mathrm{pH}$ ranged from 6.0 to 8.5 by urine dipstick for all measurements, which was within the recommended
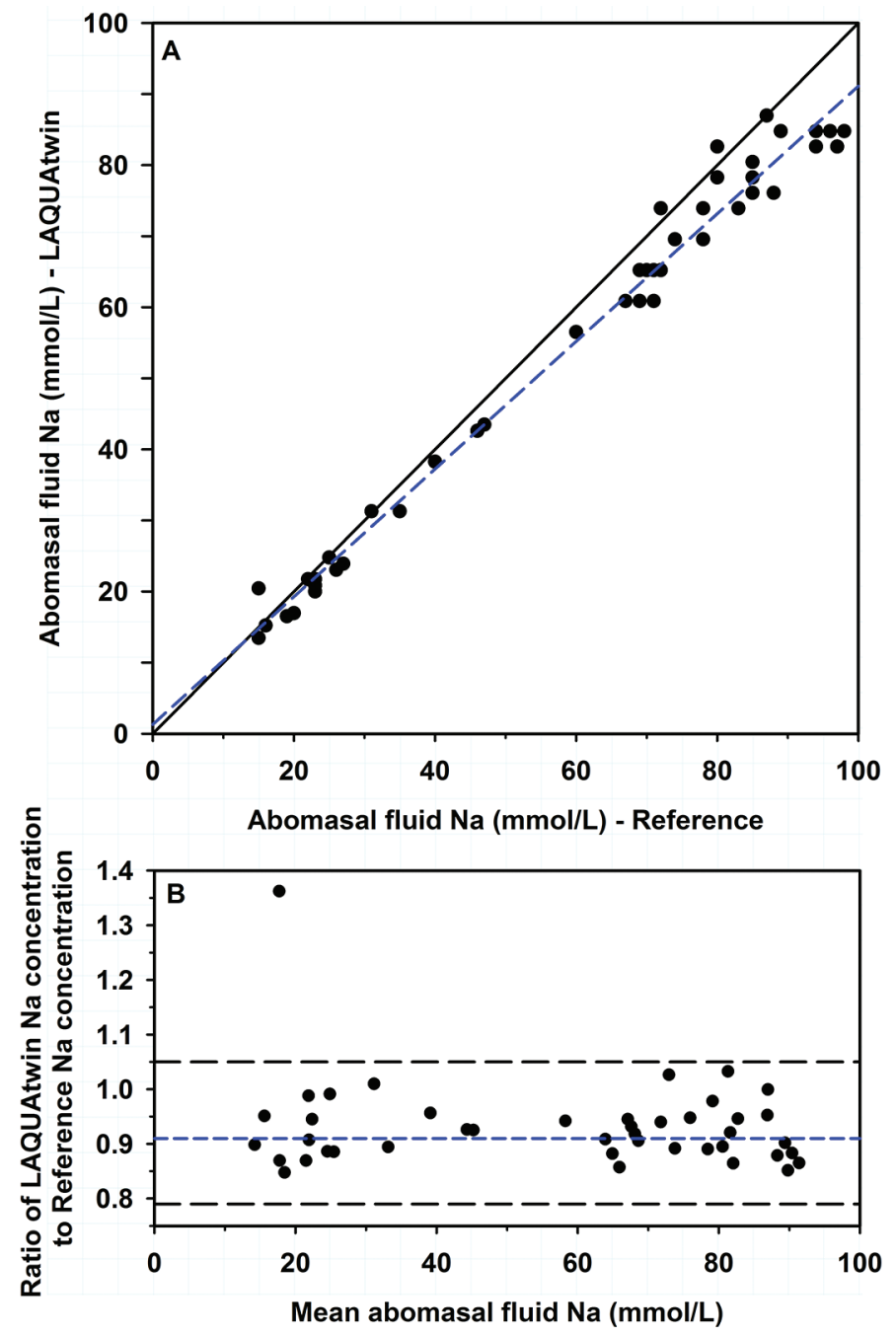

Figure 5. (A) Scatterplot indicating the relationship between the abomasal fluid sodium concentration ([Na]) measured by a direct ionselective electrode (ISE) method (LAQUAtwin $\mathrm{Na}^{+}$meter; Horiba Instruments Inc., Irvine, CA) versus the abomasal fluid [Na] measured by an indirect ISE reference method (1:31 dilution in distilled water). Abomasal fluid samples $(\mathrm{n}=67)$ were obtained periodically from 7 Holstein-Friesian bull calves with an abomasal cannula after they suckled fresh cow milk that clotted in their abomasum. Some points are superimposed because the meter has a resolution of $4.3 \mathrm{mmol} / \mathrm{L}$ when $[\mathrm{Na}]>43 \mathrm{mmol} / \mathrm{L}$. The solid diagonal line is the line of identity, and the blue dashed line is the line of best fit from Deming regression. (B) Bland-Altman mean difference plot. The horizontal blue line with short dashes represents the mean bias, and the horizontal lines with long dashes reflect the $95 \%$ limits of agreement. The plot indicates that the mean ratio of abomasal fluid [Na] measured by the ISE meter run in direct mode to abomasal fluid [Na] measured by the reference method was 0.91 .

$\mathrm{pH}$ range for the ISE meter. Deming regression for urine $[\mathrm{Na}]$ measured by the meter used in an indirect mode against urine $[\mathrm{Na}]$ measured by the indirect ISE reference method for 206 urine samples indicated a linear relationship that was similar to the line of identity. 
The proportional bias $(0.97 ; 95 \%$ CI: 0.92 to 1.02$)$ did not differ from 1 , and the constant bias $(1.3 \mathrm{mmol} / \mathrm{L}$; 95\% CI: -0.3 to 3.0 ) did not differ from 0 (Figure 6A).

The Bland-Altman plot indicated that the bias for the ISE meter run in indirect mode was $-0.1 \mathrm{mmol} / \mathrm{L}$ compared with the indirect reference method. The $95 \%$
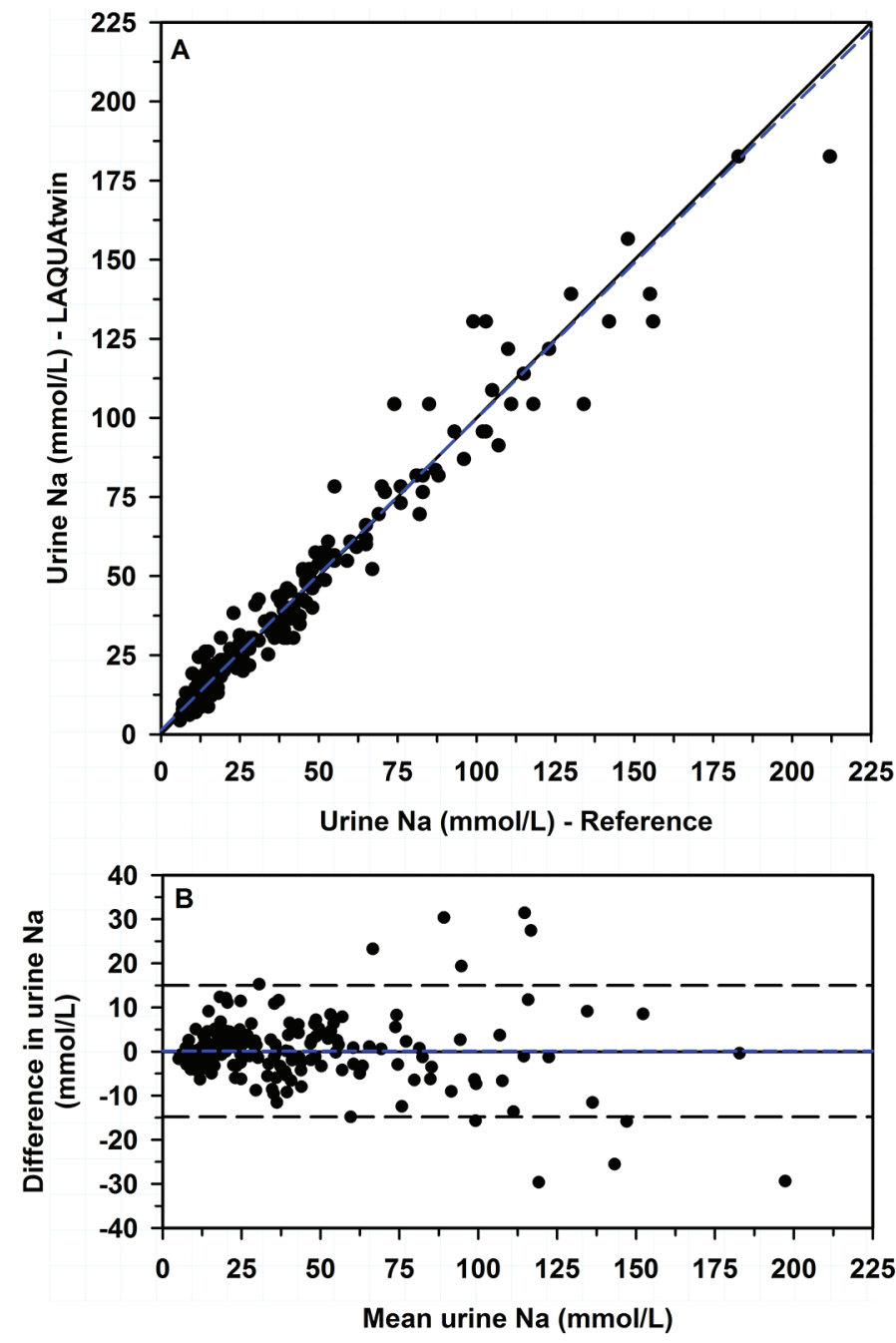

Figure 6. (A) Scatterplot indicating the relationship between the urine sodium concentration ([Na]) measured by an ion-selective electrode (ISE) method (LAQUAtwin $\mathrm{Na}^{+}$meter; Horiba Instruments Inc., Irvine, CA) run in an indirect mode (1:19 dilution in distilled water) versus the urine $[\mathrm{Na}$ ] measured by an indirect ISE reference method (1:31 dilution in distilled water). Urine samples $(\mathrm{n}=206)$ were obtained from 16 lactating Holstein-Friesian cows with experimentally induced electrolyte and acid-base imbalances. The solid diagonal line is the line of identity, and the blue dashed line is the line of best fit from Deming regression. (B) Bland-Altman mean difference plot. The horizontal blue line with short dashes represents the mean bias, and the horizontal lines with long dashes reflect the $95 \%$ limits of agreement. The plot indicates that the ISE meter run in indirect mode measured urine $[\mathrm{Na}$ ] similarly to the indirect reference method, with a mean bias of $0.1 \mathrm{mmol} / \mathrm{L}$ that was not significantly different from 0 , and limits of agreement from -14.8 to $15.0 \mathrm{mmol} / \mathrm{L}$.
LoA ranged from -14.8 (95\% CI: -18.4 to -12.3$)$ to 15.0 (95\% CI: 12.5 to 18.6$) \mathrm{mmol} / \mathrm{L}$. Inspection of the plot indicated that the differences were homoscedastic and that approximately $95 \%$ of the data points were within the LoA (Figure 6B). Proportional error was not evident in the plot. The $95 \%$ CI for LoA was 37.0 $\mathrm{mmol} / \mathrm{L}$. In summary, the bias was approximately 0 , but the $95 \%$ CI for the LoA exceeded the a priori defined range of $\pm 4 \mathrm{mmol} / \mathrm{L}$.

\section{DISCUSSION}

This appears to be the first study to characterize the analytical performance of the portable LAQUAtwin ISE meter for measuring $[\mathrm{Na}]$ in biological fluids from cattle or other domestic animals. The Bland-Altman plot indicated that the ISE meter run in direct mode measured whole-blood [Na] $4.7 \mathrm{mmol} / \mathrm{L}(3.8 \%)$ higher than the corresponding plasma sample (Figure 2B). The magnitude of the difference approximated the 3.0-mmol/L difference recently reported when human whole-blood and plasma $[\mathrm{Na}]$ were measured by the same direct ISE analzyer (Langelaan et al., 2017). Differences occur when using ISE technology to analyze whole-blood samples because the saturated hypertonic potassium chloride bridge and resultant hypertonic solution between the measuring and reference electrode causes localized dilution of plasma around the measuring electrode as water moves from inside the erythrocyte to plasma, thereby creating a residual junction potential (Bijster et al., 1983; Langelaan et al., 2017). The residual junction potential is usually positive and can increase the measured electromotive force in whole blood by up to $0.6 \mathrm{mV}$ (Langelaan et al., 2017), thereby increasing the calculated value for $[\mathrm{Na}]$ in whole blood.

The Bland-Altman plot indicated that the LAQUAtwin ISE meter had a constant bias of $-9.7 \mathrm{mmol} / \mathrm{L}$ $(-7.3 \%)$ in the measured value for whole-blood [Na], compared with the Rapidpoint 405 blood analyzer value (Figure 1B). The 1988 United States Clinical Laboratory Improvement Amendments recommended a maximum difference in measured [Na] of $4.0 \mathrm{mmol} / \mathrm{L}$ (Ehrmeyer et al., 1990). The mean difference in measured [Na] value was most likely due to the presence of a built-in conversion algorithm in the Rapidpoint 405 blood analyzer, which ensured that the result was comparable to flame photometry of a plasma sample with typical plasma protein concentration of $72 \mathrm{~g} / \mathrm{L}$ after adjustment for the residual junction potential (D'Orazio et al., 2000). Our method comparison results therefore suggest that LAQUAtwin whole-blood [Na] measurements should be routinely increased by $7.3 \%$ to provide a clinically useful value for whole-blood [Na]. 
The Bland-Altman plot indicated that the LAQUAtwin ISE meter run in direct mode had a constant bias of $-16.7 \mathrm{mmol} / \mathrm{L}(-12.7 \%)$ in the measured value for plasma [Na], compared with the plasma $[\mathrm{Na}]$ measured by the Cobas c 311 analyzer employing an indirect ISE method (Figure 3B). In other words, LAQUAtwin plasma $[\mathrm{Na}]$ measurements should be routinely increased by $12.7 \%$ to provide a clinically useful value for plasma $[\mathrm{Na}]$.

The blood-milk barrier is damaged in cattle with subclinical and clinical mastitis, causing the tight junctions between glandular secretory cells to become leaky, thereby promoting the movement of extracellular fluid components, including $\mathrm{Na}$ and $\mathrm{Cl}$, into the lumen of the alveolus, where they mix with milk and increase the $\mathrm{Na}$ and $\mathrm{Cl}$ concentration (Zhao and Lacasse, 2008). The Bland-Altman plot indicated that the LAQUAtwin ISE meter run in direct mode had a constant bias of $-3.1 \mathrm{mmol} / \mathrm{L}(-15.0 \%)$ in the measured value for milk [Na], compared with the milk [Na] measured by the reference method (Hitachi 917) employing an indirect ISE method (Figure 4B). This means that LAQUAtwin milk $[\mathrm{Na}]$ measurements should be routinely increased by $15.0 \%$ to provide a clinically useful value for plasma $[\mathrm{Na}]$.

The Bland-Altman plot indicated that the LAQUAtwin ISE meter run in direct mode had a constant bias of $-9.0 \%$ in the measured value for abomasal fluid [ $\mathrm{Na}$, compared with the abomasal fluid $[\mathrm{Na}]$ measured by the Hitachi 911 analyzer employing an indirect ISE method (Figure 5B). LAQUAtwin plasma [Na] measurements should therefore be routinely increased by $9.0 \%$ to provide a clinically useful value for abomasal fluid [Na]. Finally, our findings indicated that the measured [Na] does not need to be adjusted when urine is analyzed by the LAQUAtwin ISE meter in an indirect mode.

The major limitation of the LAQUAtwin ISE meter was that the measurement resolution changes across 3 ranges: $0.043 \mathrm{mmol} / \mathrm{L}$ up to $4.3 \mathrm{mmol} / \mathrm{L} ; 0.43 \mathrm{mmol} / \mathrm{L}$ from 4.3 to $43 \mathrm{mmol} / \mathrm{L}$; and $4.3 \mathrm{mmol} / \mathrm{L}$ from 43 to $430 \mathrm{mmol} / \mathrm{L}$. The measurement resolution is adequate for clinical use, but the results of the study reported here do not support use of the meter in research studies where whole-blood, plasma, or serum $[\mathrm{Na}]$ is measured. Improved measurement resolution, particularly over the range of 43 to $430 \mathrm{mmol} / \mathrm{L}$, and including this range of values within the recommended range of use, would likely improve the clinical utility of the meter for diagnosing hyponatremia in critically ill calves and adult cattle.

We conclude that the portable LAQUAtwin ISE $\mathrm{Na}$ meter provides a sufficiently reliable and low-cost cow-side instrument for measuring $[\mathrm{Na}]$ in whole-blood, plasma, milk, abomasal fluid, and urine samples in cattle and is suitable for clinical use when some degree of imprecision is acceptable. It should be noted that the meter is not approved for medical use by the Food and Drug Administration in the United States. The recommended measurement range $(0.043$ to $99 \mathrm{mmol} / \mathrm{L})$, variable resolution throughout the measurement range, and large LOA with reference methods negatively affect the applicability of the LAQUAtwin ISE Na meter for research purposes.

\section{ACKNOWLEDGMENTS}

The authors thank the staff at the Clinic for Ruminants, LMU Munich (Oberschleißheim, Germany), and the staff at the Clinical Pathology Laboratory, College of Veterinary Medicine, at the University of Illinois at Urbana-Champaign, for their assistance. Funding for this study was provided, in part, by a Government Mission Program grant from the Cultural and Educational Bureau, Embassy of the Arab Republic of Egypt (A. A. Megahed), the Scholarship Division, Ministry of Education, Malaysia (M. W. H. Hiew), and a research grant (TR 1321/1-1) from the German Research Foundation (Deutsche Forschungsgemeinschaft, Bonn, Germany; F. M. Trefz).

\section{REFERENCES}

Bijster, P., H. L. Vader, and C. L. J. Vink. 1983. Influence of erythrocytes on direct potentiometric determination of sodium and potassium. Ann. Clin. Biochem. 20:116-120.

Bland, J. M., and D. G. Altman. 2007. Agreement between methods of measurement with multiple observations per individual. J. Biopharm. Stat. 17:571-582.

Brooks, C. L., F. Garry, and M. S. Swartout. 1988. Effect of an interfering substance on determination of potassium by ion-specific potentiometry in animal urine. Am. J. Vet. Res. 49:710-714.

Burnett, R. W., A. K. Covington, N. Fogh-Andersen, W. R. Külpmann, A. Lewenstam, A. H. Maas, O. Müller-Plathe, C. Sachs, O. Siggaard-Andersen, A. L. VanKessel, and W. G. Zijlstra. 2000. Recommendations for measurement of and conventions for reporting sodium and potassium by ion-selective electrodes in undiluted serum, plasma or whole blood. Clin. Chem. Lab. Med. 38:10651071.

Chhapola, V., S. K. Kanwal, and R. Brar. 2015. Reporting standards for Bland-Altman agreement analysis in laboratory research: A cross-sectional survey of current practice. Ann. Clin. Biochem. $52: 382-386$.

Constable, P. D., W. Grunberg, and L. Carstensen. 2009. Comparative effects of two oral rehydration solutions on milk clotting, abomasal luminal $\mathrm{pH}$, and abomasal emptying rate in suckling calves. J. Dairy Sci. 92:296-312.

Constable, P. D., M. W. H. Hiew, S. Tinkler, and J. Townsend. 2014. Efficacy of oral potassium chloride administration in treating lactating dairy cows with experimentally induced hypokalemia, hypochloremia, and alkalemia. J. Dairy Sci. 97:1413-1426.

D'Orazio, P., W. G. Miller, G. L. Myers, D. T. Basil, J. H. Eckfeldt, S. A. Evans, G. A. Graham, P. J. Parsons, and N. V. Stanton. 2000 Standardization of sodium and potassium ion-selective electrode systems to the flame photometric reference method; Approved 
Standard-Second Edition, C29-A2 [electronic document]. CLSI. 20:1-22.

Dimeski, G., and R. J. Barnett. 2005. Effects of total plasma protein concentration on plasma sodium, potassium and chloride measurements by an indirect ion selective electrode measuring system. Crit. Care Resusc. 7:12-15.

Dimeski, G., P. Mollee, and A. Carter. 2006. Effects of hyperlipidemia on plasma sodium, potassium, and chloride measurements by indirect ion selective electrode measuring system. Clin. Chem. $52: 155-156$.

Dimeski, G., T. J. Morgan, J. J. Presneill, and B. Venkatesh. 2012. Disagreement between ion selective electrode direct and indirect sodium measurements: Estimation of the problem in a tertiary referral hospital. J. Crit. Care 27:326.e9-326.e16.

Ehrmeyer, S. S., R. H. Laessig, J. E. Leinweber, and J. J. Oryall. 1990 1990 Medicare/CLIA final rules for proficiency testing: Minimum intralaboratory performance characteristics (CV and bias) needed to pass. Clin. Chem. 36:1736-1740.

Giavarina, D. 2015. Understanding Bland Altman analysis. Biochem. Med. (Zagreb) 25:141-151.

Harr, K. E., B. Flatland, M. Nabity, and K. P. Freeman. 2013. ASVCP guidelines: Allowable total error guidelines for biochemistry. Vet. Clin. Pathol. 42:424-436.

Jain, A., I. Subhan, and M. Joshi. 2009. Comparison of the point-ofcare blood gas analyzer versus the laboratory auto-analyzer for the measurement of electrolytes. Int. J. Emerg. Med. 2:117-120.
Jensen, A. L., and M. Kjelgaard-Hansen. 2006. Method comparison in the clinical laboratory. Vet. Clin. Pathol. 35:276-286.

Langelaan, M. L., L. Kamp, E. Zandijk, and M. T. Raijmakers. 2017. Prevalence of pseudonatremia in a clinical laboratory-Role of the water content. Clin. Chem. Lab. Med. 55:546-553.

Martin, R. F. 2000. General Deming regression for estimating systematic bias and its confidence interval in method-comparison studies. Clin. Chem. 46:100-104.

Megahed, A. A., M. W. H. Hiew, W. Grünberg, and P. D. Constable. 2016. Evaluation of 2 portable ion-selective electrode meters for determining whole blood, plasma, urine, milk, and abomasal fluid potassium concentrations in dairy cattle. J. Dairy Sci. 99:73307343.

Olofsen, E., A. Dahan, G. Borsboom, and G. Drummond. 2015. Improvements in the application and reporting of advanced BlandAltman methods of comparison. J. Clin. Monit. Comput. 29:127139.

Zhao, X., and P. Lacasse. 2008. Mammary tissue damage during bovine mastitis: causes and control. J. Anim. Sci. 86:57-65.

Zou, G. Y. 2013. Confidence interval estimation for the Bland-Altman limits of agreement with multiple observations per individual. Stat. Methods Med. Res. 22:630-642. 